\title{
On the Contemporary Practice of Philosophy of Mathematics
}

\author{
Colin Jakob Rittberg \\ Centre for Logic and Philosophy of Science, \\ Vrije Universiteit Brussel \\ Pleinlaan 2, \\ Brussels 1050, Belgium \\ E-mail: colin.jakob.rittberg@vub.be
}

\begin{abstract}
In this paper I propose to reflect on contemporary philosophy of mathematics. I argue that the impartiality to methodology that is present in the current practice of philosophy of mathematics is an intellectual vice of our research community because it leaves our philosophical methods to engage with mathematics implicit. This hides difficulties our research community faces, difficulties our students can reasonably expect to learn about and which we need to engage with to overcome them. To be virtuously open-minded about methodology is not to be impartial about methodology but rather to critically assess the proposals on offer in a public debate.
\end{abstract}

Keywords: indifference, methodology, open-mindedness, philosophy of mathematical practice, tolerance, virtue

\section{Introduction}

The philosophy of mathematics is not a homogeneous whole. The common story is that there is a mainstream philosophy of mathematics and a diverging trend, the so-called philosophy of mathematical practice. The former is concerned with issues surrounding the ontology of mathematics and the foundational debate. What the latter is concerned with is less clear. Following the inclusive lines of the Association for the Philosophy of Mathematical Practice, it includes all philosophical works about mathematics which do not fit under the 'mainstream' heading. 
This split of the philosophy of mathematics can be traced back to Aspray's and Kitcher's 'Opinionated Introduction' (1988). They individuate two traditions which they call 'orthodox' and 'maverick'. For them, the orthodox tradition focusses mainly on the metaphysical problems surrounding mathematics. The maverick tradition is, according to Aspray and Kitcher, championed by Lakatos and is concerned with a philosophical engagement of the methodology of mathematics. ${ }^{1}$

Twenty years later, in the introduction to his book, Mancosu (2008) argues that the philosophy of mathematics has developed further into three traditions: the mavericks on the one side, the orthodox or, as he now calls them, mainstream tradition on the other and somewhere in between is what he calls 'the philosophy of mathematical practice'. Unlike the mavericks, which have, according to Mancosu (2008, p. 5), an "iconoclastic attitude" with respect to mainstream philosophy of mathematics, Mancosu's philosophers of mathematical practice do not wish to criticise the mainstream tradition. Instead, they claim that some issues are worthy of philosophical attention which thus far have been ignored by the mainstream tradition, such as "fruitfulness, evidence, visualization, diagrammatic reasoning, understanding, explanation and other aspects of mathematical epistemology which are orthogonal to the problem of access to 'abstract objects'” (Mancosu, 2008, pp. 1-2). For Mancosu, the mavericks were rebels who wanted to change how philosophy of mathematics is done. Mancosu's philosophers of mathematical practice, by contrast, wish only to extend the scope of the philosophical questions asked about mathematics; they do not challenge the mainstream tradition.

Mancosu's terminology is, in light of contemporary usage, somewhat unhappy. For Mancosu there is a difference between the mavericks and the philosophers of mathematical practice; Lakatos is a maverick and not a philosopher of mathematical practice on Mancosu's account. This conflicts with the widespread narrative of Lakatos as the founding father of philosophy of mathematical

\footnotetext{
Authors who have subsequently repeated Aspray's and Kitcher's narrative, such as Mancosu (2008) and Carter (2019), have often failed to mention what Aspray and Kitcher readily admit, namely "a bias toward English language literature and to research conducted by mathematicians and historians of the United States" in their presentation of the state of the field (Aspray \& Kitcher, 1988, p. 20). Such authors tacitly excuse Aspray's and Kitcher's bias rather than making it part of the narrative, thereby deforming what we can (and should) learn from Aspray and Kitcher.
} 
practice. ${ }^{2}$ I will use the more common conception in this paper, referring to 'Mancosu's philosophy of mathematical practice' when necessary.

Aspray and Kitcher (1988) had presented the practice-based approaches to philosophy of mathematics as a largely unified camp. By 2008, Mancosu already saw a divide. In 2014, Van Bendegem tells us that there are many more divisions within this camp. Van Bendegem (2014) distinguishes eight partly overlapping approaches to a practice-based philosophy of mathematics. The Lakatosian approach focusses on processes in mathematics rather than final mathematical products. The descriptive analytical naturalising approach aims to explicate mathematical concepts such as 'proof' by describing how they are used by the mathematicians. The normative analytical naturalising approach explicates mathematical concepts by subjecting studies on how mathematicians use these concepts to philosophical criticism. The sociology of mathematics focusses mainly on the community or group phenomena found in mathematical practices. Educationalists take seriously the idea that for mathematical communities to form, agents need to be educated - this forges links with the works of the mathematics educators. The ethnomathematics approach stresses that mathematical practices are culturally embedded, which creates ties to the work done on ethnomathematics. Evolutionary biology of mathematics inquires how mathematical knowledge is biologically encoded in our human body. Studies in the cognitive psychology of mathematics aim to understand the psychological influences on mathematical thinking.

Carter (2019) also highlights the heterogeneous nature of the practice-based camp. She identifies three, sometimes overlapping, strands: an agent-based, a historical and an epistemological strand. Two of these strands Carter distinguishes further into different flavours. The agent-based strand holds that a philosophy of mathematics should (or has to) take the human being doing mathematics into account. According to Carter, there are two flavours. One views mathematics as a fundamentally social activity and has strong ties to sociology. The other, Carter calls it the 'pragmatic' flavour, is mainly concerned with an agentbased understanding of mathematical knowledge. The historical strand in the philosophy of mathematical practice is, according to Carter, mainly concerned with the fact that mathematics has a history and the philosophical issues arising from it. Mathematics is viewed as "the product of certain activities and not a

2 For Lakatos as a founding father see, e.g., Van Bendegem (2014). For a counter-argument to the Lakatos-as-the-founding-father narrative see Rittberg (2018). For use of Mancosu's terminology see, e.g., Cellucci (2017). 
static theory" (Carter, 2019, p. 11). Carter discusses three flavours of this. One aims to reveal the philosophical issues in mathematical development. Another investigates the philosophical beliefs of the mathematicians themselves. The third aims to develop "philosophical tools in order to understand the history of mathematics". The last strand, epistemological philosophy of mathematical practice, aims to answer traditional questions, such as about "the nature of mathematical objects and what constitutes our knowledge of them" (Carter, 2019 , p. 16), by engaging with "real mathematics" (Corfield, 2003) and "not some idealised notion of the discipline" (Leng, 2002).

These survey articles reveal that the philosophy of mathematical practice is not homogeneous. The term 'philosophy of mathematical practice'4 provides a forum to discuss a plethora of philosophical questions and approaches to the philosophy of mathematics. On the upside of this, no voice needs to go unheard just because it does not fit within some pre-established boxes. The downside is that throwing all those philosophical works on mathematics which do not fit into the mainstream box into the practice-based box discourages critical methodological assessment within this box. Carter (2019) gets at this when she correctly presents the philosophy of mathematical practice as a loosely connected but partly disjointed enterprise.

Because attention to "[mathematical] practice is to a greater or lesser extent on the agenda of basically all active philosophers of mathematics" (Ferreirós \& Gray, 2006, p. 12), the line between mainstream and practice-based philosophy of mathematics is blurry at best. We should ask ourselves how useful the mainstream/ practice-based terminology is to talk about our field. It is my contention that the distinction is useful as a means to organise the field, precisely because the inherent vagueness of 'practice-based approach' avoids boxed-in thinking. As a philosophical tool to think about the field, however, it has become unsuitable. The term 'philosophy of mathematical practice' suggests dividing lines where there may not be any (cf. Ferreirós \& Gray, 2006) and fails to draw attention to the marked differences of approaches in the field (Carter, 2019). For this

3 Recall here Lakatos' paraphrase of Kant: "the history of mathematics, lacking the guidance of philosophy, has become blind” (Lakatos, 1976, p. 2).

4 There is some disagreement whether one should speak of the singular practice or the plural practices when speaking of the philosophy of mathematical practice[s]. This may depend on whether practice is a mass- or a count-noun. But the term 'practice' may also be like the term 'water': a mass noun where it can make sense to add the 's' to speak of treacherous waters, for example. My personal preference is to speak of a philosophy of mathematical practices in this water-like sense. In this section, however, I avoid using the 's' to align with the terminology of Mancosu (2008) and Carter (2019). 
reason, I will not use the term 'philosophy of mathematical practice' in what follows. I will use 'philosophy of mathematics', by which I intend any kind of philosophical work about mathematics, practice-based or otherwise. That said, my examples are taken mostly from philosophical works that engage with mathematics through case studies of its practice. This is because of my personal interest in (and familiarity with) such works.

Aspray's and Kitcher's, Mancosu's, Van Bendegem's, and Carter's discussions of the philosophy of mathematics all individuate the various traditions, approaches or strands in the philosophy of mathematics by their aims and questions. ${ }^{5}$ What methods these philosophers use to answer their questions remains secondary. The spirit of especially Mancosu's and Carter's work is to allow any kind of reasonable method, whereby 'reasonable' remains unstated and unclarified. I argue in this paper that in its current form, this kind of impartiality to the methods used (a) fails to realise that the different philosophical methods employed have led to conflicting results and (b) does not provide sufficient methodological guidelines on how philosophy of mathematics is and should be done. What we need, so is the claim of this paper, is a debate about the methods of philosophy of mathematics.

\section{A first example of conflicting methods}

Mathematical proofs are not formal derivations. A formal derivation is defined according to the strict definition of 'proof' as used in Hilbert-style proof-theory. The kind of proofs one finds in mathematical journals, I call them mathematical proofs, differ from formal derivations. They employ natural language and/or pictures, have gaps and so on. ${ }^{6}$

According to a widely spread assumption, mathematical proofs merely help to facilitate communication. The epistemologically relevant parts of a mathematical proof are all captured by its formal derivation. ${ }^{7}$ One of the staple results of

5 Other noteworthy survey papers that could not be engaged with here due to limitations of space include Löwe (2016), Giardino (2017), Löwe \& Van Kerkhove (2019), and Hamami \& Giardino (forthcoming).

6 Mathematical proofs have become a subject of study: Manders (2008), Tanswell (2015),

Andersen (2017a; 2018), Andersen et al. (2019), Sørensen et al. (2019).

7 See, for example, Feferman's Formalisability Thesis (2012). 
the philosophy of mathematical practice is that this assumption is incorrect. ${ }^{8}$ Should mathematical proofs then not replace the formal derivations in our epistemology of mathematics? Those who already think that we should "study what mathematicians actually do" and "take mathematical practice seriously" will readily agree. But why? The idea seems to be that here we have some description of what mathematical practice is like and we should account for that. "No idealisation!" was the rallying cry we might take from Leng's quotation given above. But philosophers idealise. ${ }^{10}$ Much of philosophy aims to give answers to general questions, and to do this philosophers have to move away from the specifics. They thus have to ensure that their idealisations are not philosophically damaging, rather than to avoid idealisations entirely. In what way is it philosophically damaging to assume that mathematical proofs are essentially formal derivations? This points towards a possible defence for the formal derivationist's position. From Leitgeb (2009) we can learn, the formal derivationists might argue, that an understanding of mathematical reasoning which does not replace mathematical proofs with formal derivations is thus far lacking. To fully replace our formal derivation account of mathematical proofs in our epistemology of mathematics thus demolishes our accounts thereof. It is better, so the argument of the formal derivationist could continue, to have an epistemological account, even if it might be idealised and perhaps even philosophically damaging at times, than to have no epistemological account at all. Thus, the formal derivationists might conclude, mathematical proofs should not replace formal derivations in our epistemological accounts of mathematics. ${ }^{11}$ In fact, naïve derivationist's accounts, which take mathematical proofs to be formal derivations, have been overcome by more sophisticated versions which assume that the mathematical proof sketches (MacLane, 1986), indicates (Azzouni, 2004) or is a recipe or description for obtaining (Avigad, 2005) the formal derivation. Such sophisticated versions of derivationism aim to cater to the demand that

8 See, for example, Van Bendegem (1988; 1990), Rav (1999), Larvor (2008), Löwe \& Müller (2008), Mancosu (2008), Tanswell (2015).

9 These are two mantras one often hears from so-called philosophers of mathematical practice(s).

10 I do not wish to suggest here that Leng thinks otherwise. Rather, I use the quotation from Leng as a rhetorical device to draw attention to the fact that philosophy, by its nature, idealises.

11 Somewhat ironically, the formal derivationists find support for the above-presented argument in Lakatos, the arch-maverick in Aspray's and Kitcher's as well as Mancosu's book. Lakatos (1978) has convincingly argued that a fallibilism which discards our current theory as soon as it is falsified is naïve. Falsified theories are only discarded once a better one is at hand to replace it. Philosophers of mathematics wishing to replace the derivationist account, as the formal derivationists could claim, still need to provide such a better theory. Larvor (2017) takes this line of reasoning seriously and explores the possibility of extending a narrow epistemic model of informal proofs to a wider range. 
philosophy should not idealise too much whilst still providing an answer to philosophical riddles such as "what is validity?". ${ }^{12}$

In this section, I have rehearsed a well-known debate in the philosophy of mathematics with the aim of making visible how different methods are used and have led to conflicting results in the philosophy of mathematics. It may however be argued that derivationism and non-derivationism are philosophical views rather than methods. This would mean my example would be ill chosen.

Is derivationism a method or a philosophical view? In the philosophical debate about what ensures the validity of a mathematical proof, derivationism provides an answer. Another answer is currently under development by the non-derivationists (e.g., Larvor, 2017). In this light, derivationism seems more a view than a method because it is a position one might take in the ongoing philosophical debate about what ensures the validity of a mathematical proof. On the other hand, to embrace derivationism entails (or presupposes) a willingness to abstract away from the proofs as they present themselves in mathematical practice. The non-derivationist also allows such abstraction but aims to remain closer to mathematical practice than the derivationist. How far one allows oneself to abstract away from the subject of one's study is, however, more a method than a view. That is, in this light derivationism is a method rather than a view. And this makes visible the entanglement between our methods and our views. We may hold certain views because we deem certain methods (non-)admissible just as we may be led to embrace certain methods because of the views we hold. The derivationism/non-derivationism debate is thus neither solely about methods nor solely about views: it is about both.

Derivationism provides a widely accepted epistemological narrative with answers to philosophical questions such as "What is validity?" or "Why is mathematical knowledge epistemologically special?". Non-derivationism distorts less our view on mathematics as performed by the mathematicians. On its own, this does not suffice as an argument for either approach. Currently, both are in use

12 The debate described above is about how proofs in mathematics work. There is also a debate about what should count as a proof in mathematics. Brouwer, Prawitz, Martin-Löf, and others have proposed constructivist accounts of proof which challenge the Hilbert-style formal derivation discussed above. There are points of contact between the debate on how proofs work and the debate about what should count as proof. Gentzen (1935) viewed his Natural Deduction as natural because of its (alleged) closeness to mathematical practice. Voevodsky's Univalent Foundation axiom formalises, according to the authors of HoTT (2013, p. 6), a certain abuse of notation common in mathematical practice. A closer study of these matters is beyond the scope of this paper. 
side-by-side in the philosophy of mathematics. This introduces an incoherence into our philosophical accounts of mathematics. Van Bendegem made a similar point when he wrote about the heterogeneity of approaches in the philosophy of mathematics:

One might think that perhaps we are dealing here with a division of labour of a vast field to explore, but such a division suggests that all the parts can be put together again to form a minimally coherent whole. And that is (at present) definitely not the case. (Van Bendegem, 2014, p. 222)

Incoherence within a research tradition can lead to progress because it invites the researchers to overcome the incoherence. Incoherence can lead to disagreement, such as, for example, over whether this or that philosophical method delivers a satisfactory philosophical account in response to a given question. Virtuously handled, such disagreement is positive. It can inspire argument and research which ultimately can lead to progress. For this to happen, for incoherence to lead to virtuous disagreement and progress, the incoherence needs to be recognised as such. To deem the involved conflicting positions to be simply different schools of thought which should be allowed to co-exist side-by-side is an ad hoc solution which side-steps the problem; this is indifference rather than tolerance. ${ }^{13}$ Instead, the incoherence within a research tradition should be critically assessed with the aim to overcome it. It may still turn out that there really are different schools of thoughts in play, all of which have their own merits. But in this case, there would be an argument why the incoherence exists and is acceptable in the research community, rather than the mere ad hoc solution of deeming each opposing position worthy before critical assessment.

\section{Practices: how to find out?}

An implicit assumption in the above point about proofs is that it is a fact of the matter about mathematical practices that mathematicians do not write down proofs as formal derivations. This point has been amply argued for and there is little reason to doubt it. ${ }^{14}$ The larger question is however how one can find out whether something is a fact of the matter about mathematical

13 From Valitova $(1998$, p. 23) we can learn that "tolerance involves an interested relation to the other". On indifference, see Lillehammer (2014a, b).

14 See Van Bendegem (1988; 1990), Rav (1999), Larvor (2008), Löwe \& Müller (2008), Mancosu (2008), Tanswell (2015). 
practice. This question is hardly ever addressed in the works on the philosophy of mathematics. ${ }^{15}$

Here is a list of methods that have been used by philosophers of mathematics to find out about mathematical practice: ${ }^{16}$

1. Reading the works of mathematicians: e.g., reading technical mathematical works (Larvor, 2010); reading non-technical works (Rittberg et al., 2018); corpus analysis (Johansen et al., 2018).

2. Historical approaches: e.g., accessing philosophical questions through previous historical and/or biographical work (Andersen, 2017b); accessing social practices of mathematics through historical sources (Gerovitch, 2016).

3. Interviews with mathematicians: e.g., structured interviews (Weber, 2008); unstructured interviews (Maddy, 1988a, b).

4. Anthropological approaches: e.g., embedding with mathematicians (Kaufman, 2016); longitudinal studies (Andersen et al., 2019); observations of mathematical activity (Barany \& MacKenzie, 2014); analysis of internet resources (Martin, 2015); ethnomathematics (François \& Vandendriesche, 2016).

5. Quantitative empirical research: e.g., questionnaire studies (Inglis \& Aberdein, 2014).

6. Report of experiences: e.g., collaboration with mathematicians (De Toffoli \& Giardino, 2014); reporting one's own mathematical research experience (Rav, 1999).

7. Cognitive science: e.g., theoretical studies (Núñez, 2005); empirical studies (van der Ham et al., 2017).

Methods to engage with a practice are not unproblematic. Philosophers of science know this. Pitt (2008), for example, speaks of a "dilemma of case studies", in which we either start with our philosophical view, thereby risking to cherry-pick case studies that support our views, or start with the case studies and then face an inductive fallacy when aiming to say something general. ${ }^{17}$ Besides these general worries, the individual methods face problems of their own. Social scientists know this. They write books about, for example, how interviews should be conducted. In the philosophy of mathematics, however, there is very

15 Notable exceptions include Löwe et al. (2010) and Löwe \& Van Kerkhove (2019).

16 This list has been compiled in joint work with Henrik Kragh Sørensen. It was originally published in Rittberg \& Van Kerkhove (2019).

17 For answers to this dilemma see Burian (2001; 2002), Schickore (2011), Chang (2011), Scholl \& Räz (2016), Rittberg \& Van Kerkhove (2019). 
little discussion about methods, especially in print. Young researchers are left to find out for themselves. These are the methods of their trade. They are neither obvious, nor unproblematic, nor should young researchers be stuck with the opinions of their academic tutors. Young researchers can reasonably expect to be taught the basics of their field. The philosophy of mathematics needs a body of knowledge which critically assesses our philosophical methods (to engage with mathematical practices and otherwise). A methodological debate about philosophy of mathematics would, with time, provide such a body of knowledge.

\section{Conflicting pictures of mathematical practice}

The point about philosophical methods to individuate features about mathematical practice can be strengthened by realising that different methods have already led to different results about what mathematical practice is like. The most well-known of these might be Lakatos' description of the dialectical movement of the concept of a polyhedron in his Proofs and Refutations and the counter-claim that this is a one-off rather than a feature of mathematical practice is general. ${ }^{18}$ More recent examples of this are the cases Inglis and Aberdein against Steiner and Rittberg against Maddy. ${ }^{19}$ Inglis and Aberdein claim that "a common methodological move made by philosophers of mathematics" is to offer an example of a piece of mathematics, assert that this piece of mathematics has a certain property and "appeal to the reader's intuitions for agreement" (Inglis \& Aberdein, 2016, p. 2). Inglis and Aberdein call such philosophers exemplar philosophers.

In Inglis and Aberdein (2016), the authors engage with Steiner's account of the explanatoriness of a proof. Feferman (1969) suggests that those proofs are more explanatory that are more general. Steiner (1978) presents a proof which is, according to him, more general yet not explanatory, relying on his readers to share his intuitions on the explanatoriness of the proof. Thus, Steiner is an exemplar philosopher in Inglis' and Aberdein's sense. Steiner gives his own characterisation of explanatoriness: an "explanatory proof makes reference to a characterizing property of an entity or structure mentioned in the theorem, such that from the proof it is evident that the result depends on the property" (Steiner,

18 Larvor (1997) engages with the one-off claim in some detail.

19 Further examples, which will not be discussed in detail here, include Friend's (2014) argument that set theorists are more pluralistic than Maddy realises. See also Rittberg \& Van Kerkhove (2019) on this point. 
1978, p. 143). Steiner supports his understanding of explanatoriness by two examples: a proof which is, according to his expert introspection, explanatory and which fits his characterisation and a proof which is, again according to him, not explanatory and which does not fit his characterisation.

In their work, Inglis and Aberdein rely on statistical methods and results. They chose a proof which had been deemed worthy of inclusion in Proofs from The Book and presented this proof in an online study. One hundred and twelve American mathematicians participated in the study. Inglis' and Aberdein's results showed a remarkable disagreement amongst mathematicians on proof-appraisal.

Steiner argues that a general account of the explanatoriness of proofs can be cashed out by relying on expert introspection (or 'intuition' as Inglis and Aberdein call it). Inglis and Aberdein use statistical methods to argue that whether a proof is seen as explanatory is largely idiosyncratic. The use of statistical methods thus leads to results which conflict with the results of a method which heavily relies on expert introspection. Steiner, Inglis and Aberdein all wish to be sensitive to mathematical practice, so whom should we follow here? Which methods do we deem acceptable and why?

Another case in point is the case Rittberg against Maddy. According to Maddy (1997; 2011), some methodological debates in set theory have been resolved but the philosophical debates have not, from which she follows that the methodological debates have not been resolved on philosophical grounds (Maddy, 1997, p. 191). She thus excludes the metaphysical views of the set theorists from her philosophical analysis of the practice, dubbing them as colourful asides which are not part of the evidential structure of the subject (Maddy, 2011, p. 53). In Rittberg (2016a, b) I argue against this. I analyse programmatic papers of two set theorists, Woodin and Hamkins, to argue that the metaphysical beliefs of these set theorists can stand in a reciprocal relationship to the way these set theorists practise set theory.

My investigation of set-theoretic practice suggests that metaphysical beliefs can play a role in mathematical reasoning practices. According to Maddy, such metaphysical beliefs are colourful asides. We both aim at describing what the philosophically relevant parts of the practice are like, but we reach different conclusions. With whom should a philosopher who wishes to take mathematical practice seriously side? 


\section{Open-mindedness}

Writing about the three main types of methodologies used in the social sciences (quantitative, qualitative and mixed methods) Symonds and Gorard write:

in the education research community we have observed both student and seasoned researchers thinking that there are either only three ways to do research or that research must align with one of these categories for it to be valid. (Symonds \& Gorard, 2010, p. 122)

Symonds and Gorard correctly point out that such thinking inhibits creativity. Hence, a methodological debate about the philosophy of mathematics should not aim to reduce the philosophy of mathematics to a few standard methodologies. Notice how this links back to the point above about disagreement potentially leading to progress. We should want different and disagreeing methodologies, because it can further the progress of the field.

Open-mindedness is an intellectual virtue. ${ }^{20}$ It encourages us to be open to the idea of multiple methodologies of the philosophy of mathematical practice being in use side-by-side. Symonds' and Gorard's can be considered as an argument for open-mindedness. But being open-minded does not mean to allow all methodologies a place at our table, to use Chang's (2012) metaphor. Openmindedness is different from what Chang calls relativism. For Chang, "relativism involves a renunciation of judgement and commitment at least to a degree, which pluralism most definitely does not" (Chang, 2012, p. 261). Open-mindedness encourages us to probe each proposed methodology without demanding that only one methodology shall be judged worthy. That is, open-mindedness encourages a pluralism about methodologies. An important difference between relativism in Chang's sense and pluralism is that the pluralist has means to block unwanted or unsuited methodologies. Not everyone is graced with a seat at our table. But this means that we need to think about which methodologies we want. When Mancosu (2008) and Carter (2019) fail to propagate the critical assessment of the methods of philosophy of mathematics, they encourage indifference rather than tolerance and open-mindedness. A virtuously open-minded approach to the philosophy of mathematics not only encourages but also critically assesses diversity in methodology.

20 To maintain focus in this paper I will treat 'virtue' as a primitive notion. For insightful discussions of the concept see Roberts \& Wood (2007). For analytical reflection and engagement with open-mindedness as an intellectual virtue see Zagzebski (1996). 
To state it clearly, I argue that we should want a variety of methodologies in the philosophy of mathematics. This we have already. Furthermore, I argue that we should critically assess these methodologies. This is currently (largely) ${ }^{21}$ lacking.

\section{A virtuous practice of philosophy of mathematics}

Just as mathematics is something that humans do, a practice, so is the philosophy of mathematics something that humans do, a practice as well. My argument is about what kind of practice the philosophy of mathematics should be. My argument has ethical undertones because it asks what kind of virtues the participants of this practice should strive to manifest. I have given some descriptions of how philosophy of mathematics is practised today in order to highlight some deficiencies it currently has. I argued that young researchers in philosophy of mathematics can reasonably expect to be taught the basics of their trade. It is thus a deficiency of the contemporary practice of philosophy of mathematics that there is currently no body of knowledge discussing these basic methods. Furthermore, I argued that narratives about the field which embrace the different methodologies on offer without critical engagement propagate indifference rather than open-mindedness. To be virtuously openminded is to critically reflect. For the practice of philosophy of mathematics to be virtuously open-minded, so I argue, we need an open and public debate about the methodologies on offer.

One might also give a functional argument here. Having a body of knowledge in which the methods of philosophy of mathematics are critically assessed will help with teaching; allows to couch funding applications into the context of an ongoing debate; may provide guidance to what papers and conferences will be of value to specific researchers; and, lest we forget, it will facilitate research by making visible the pitfalls of the various methodologies. However, in this paper I wish to concentrate on the argument about a virtuous practice of philosophy of mathematics. An elaboration on the functional argument is left for a later date.

In a virtuous practice of philosophy of mathematics there should be reflection on how its practitioners conduct research. It should allow for different approaches to provide avenues for development yet be critical enough to establish sufficient cohesion within the community. Importantly, such critical self-reflection needs

$21 \quad$ For references of exceptions, see footnote 15. 
to be public. It is not enough for the single researcher to have opinions on methodological questions. These opinions need to be made public and critically assessed by the other practitioners. Only then can the practice be said to be critically self-reflective. Such virtuous critical self-reflection, as I have aimed to make visible in this paper, is thus far lacking in the philosophy of mathematics.

\section{Becoming aware of one's own methodology}

It is essential to a public methodological debate to discuss philosophical questions as well because methods can only be critically assessed in light of what they are methods for. For example, it might be argued that Steiner (1978) aimed at providing a conceptual analysis of 'explanatoriness of a proof', whereas Inglis and Aberdein (2016) aimed at collecting sociological evidence about mathematical practice. The argument is that the methods these authors employ lead to different results because they serve to answer different questions.

We need to become aware of and discuss the aims we have in philosophising about mathematics. Do we aim to be practice-based, i.e. learning from mathematics as practised to guide our solution to philosophical puzzles? Or do we aim to be practice-oriented, i.e. critically reflect on mathematical (research?) practices with an eye towards their improvement? Or are we interested in shaping public perceptions about mathematics, perhaps puncturing holes into overly idealised views about mathematics? Or do we aim at helping with mathematics education? And once we have answered questions such as these we should provide reasons as to why our aims are worthy and how they could be achieved. Most of us are funded by public money. A simple "because I like it" is not enough. Cui bono, who benefits from the works we provide? Answers to points such as these will influence what kind of questions we ask and which methods are suitable to do so.

There is then a connection between the aims we have, the questions we ask and the methods we use. I mean all of this when I speak of methodology. Thus, a methodology includes the methods used, the questions asked, the aims had, and our reasons for such methods, aims and questions. As argued above, we should be open-minded about methodologies. That is, not only should we embrace that methodologies already differ in the aims they serve, questions they ask and methods they use, but we should actively pursue critical engagement with these elements. It is this active critical engagement with the methodologies that 
Mancosu (2008) and Carter (2019) discourage when they call for an extension of the philosophy of mathematics rather than a methodological debate.

To be aware of one's aims, to find relevant questions, to understand one's methods and to be able to provide reasons for these elements, all this is part of good philosophical practice. To call for a methodological debate may thus look like calling for good philosophical practice, which seems superfluous. The answer to this worry is that a methodological debate is public. This forces us to articulate our methodologies more clearly. Our reasons for our methodologies need to be stronger because they no longer only need to convince ourselves. Problems we have overlooked will be flagged by the participants of the debate. A methodological debate does not ensure good philosophical practice, but it contributes to it.

\section{Beginnings of a methodological debate}

Virtuous critical self-assessment entails to praise what one finds worthy about oneself. Some philosophers have already begun to publicly debate methodologies in the philosophy of mathematics. Larvor (2001) works out what a Lakatosian dialectical philosophy of mathematics might look like. More comprehensive is a methodology which is currently under development, the so-called empirical philosophy of mathematics (Buldt et al., 2008; Löwe \& Müller, 2008; 2010; Müller-Hill, 2009; Löwe et al., 2010). ${ }^{22}$ An already fully developed methodology is Maddy's Second Philosophy (Maddy, 1997; 2007; 2011).

Philosophers of mathematics might look to other disciplines for inspiration and guidance for a methodological debate about their field. The social sciences have a long tradition of debating methodological issues (the qualitative/quantitative debate) and philosophers of mathematics might learn from them. More recently, some philosophers interested in scientific practices have started to critically assess their methods. Their works will be of particular interest to a methodological debate in the philosophy of mathematics due to the ties and similarities between mathematics and the sciences. ${ }^{23}$

\footnotetext{
22 For criticism see, e.g., Pantsar (2015).

23 Examples include Rouse (1998), Soler et al. (2014), Sauer \& Scholl (2016).
} 


\section{Conclusion}

Philosophers of mathematics have learned that it is valuable to critically reflect upon the practice of mathematics. It is now time to realise that it is valuable to critically reflect upon the practice of the philosophy of mathematics as well.

\section{Acknowledgements}

This paper developed during my time at the University of Hertfordshire, and I am grateful to its members of staff and students for their insights and encouragement. I am particularly grateful to Brendan Larvor for his guidance from the early stages of this piece to the reading of draft versions. I should also like to thank the audiences in Brussels and at the Novembertagung 2016 for their insightful questions and my colleagues at the CLPS at the Vrije Universiteit Brussels for their patience and invaluable remarks over many lunch and coffee breaks. Andrei Rodin, Bart Van Kerkhove, Fenner Stanley Tanswell, Jean Paul Van Bendegem, Jip Van Besouw, Joachim Frans, Michele Friend, and Yacin Hamami have my thanks for reading drafts, deep discussions, and for the many suggestions that led to improvements of this article. I should also like to thank Amirouche Moktefi for his encouragement and two anonymous referees for their constructive criticisms. Special thanks to Pieter Present, without whom the paper would not have been published in its current form. Research for this paper was funded by the Research Foundation-Flanders (FWO), Project G056716N.

\section{References}

Andersen, L. E. (2017a), 'On the nature and role of peer review in mathematics,' Accountability in Research, vol. 24, no. 3, pp. 177-192. https://doi.org/10.1080/08989621.2016.1274885

Andersen, L. E. (2017b), 'Outsiders enabling scientific change. Learning from the sociohistory of a mathematical proof,' Social Epistemology, vol. 31, no. 2, pp. 184-191. https://doi.org/10.1080/02691728.2016.1270367

Andersen, L. E. (2018), 'Acceptable gaps in mathematical proofs,' Synthese, pp. 1-15. https://doi.org/10.1007/s11229-018-1778-8 
Andersen, L. E.; Johansen, M. W. \& Sørensen, H. K. (2019), 'Mathematicians writing for mathematicians,' Synthese, pp. 1-18. https://doi.org/10.1007/s11229-019-02145-5

Aspray, W. \& Kitcher, P., eds. (1988), History and Philosophy of Modern Mathematics, vol. 11, Minneapolis: University of Minnesota Press.

Avigad, J. (2006), 'Mathematical method and proof', Synthese, vol. 153, no. 1, pp. 105-159. https://doi.org/10.1007/s11229-005-4064-5

Azzouni, J. (2004), 'The derivation-indicator view of mathematical practice,' Philosophia Mathematica, vol. 12, no. 2, pp. 81-106. https://doi.org/10.1093/philmat/12.2.81

Barany, M. J. \& MacKenzie, D. (2014), 'Chalk: Materials and concepts in mathematics research,' in C. Coopmans, J. Vertesi, M. Lynch \& S. Woolgar (eds.) Representation in Scientific Practice Revisited, Cambridge, MA: MIT Press, pp. 107-129. https://doi.org/10.7551/mitpress/9780262525381.003.0006

Buldt, B.; Löwe, B. \& Müller, T. (2008), 'Towards a new epistemology of mathematics,' Erkenntnis, vol. 68, no. 3, pp. 309-329. https://doi.org/10.1007/s10670-008-9101-6

Burian, R. M. (2001), 'The dilemma of case studies resolved: the virtues of using case studies in the history and philosophy of science,' Perspectives on Science, vol. 9, no. 4, pp. 383-404. https://doi.org/10.1162/106361401760375794

Burian, R. M. (2002), 'Comments on the precarious relationship between history and philosophy of science,' Perspectives on Science, vol. 10, no. 4, pp. 398-407. https://doi.org/10.1162/106361402322288039

Carter, J. (2019), 'Philosophy of mathematical practice - motivations, themes and prospects,' Philosophia Mathematica, vol. 27, no. 1, pp. 1-32. https://doi.org/10.1093/philmat/nkz002

Chang, H. (2011), 'Beyond case-studies: history as philosophy' in Integrating History and Philosophy of Science, Dordrecht: Springer, pp. 109-124. https://doi.org/10.1007/978-94-007-1745-9_8

Chang, H. (2012), Is Water $\mathrm{H}_{2} \mathrm{O}$ ? Evidence, Realism and Pluralism, Boston Studies in the Philosophy of Science, vol. 293, Dordrecht: Springer Science \& Business Media.

Corfield, D. (2003), Towards a Philosophy of Real Mathematics, Cambridge: Cambridge University Press. https://doi.org/10.1017/CBO9780511487576

De Toffoli, S. \& Giardino, V. (2014), 'Forms and roles of diagrams in knot theory,' Erkenntnis, vol. 79, no. 4, pp. 829-842. https://doi.org/10.1007/s10670-013-9568-7

Feferman, S. (1964), 'Systems of predicative analysis,' The Journal of Symbolic Logic, vol. 29, no. 1, pp. 1-30. https://doi.org/10.2307/2269764

Feferman, S. (2012), 'And so on... : reasoning with infinite diagrams,' Synthese, vol. 186, no. 1, pp. 371-386. https://doi.org/10.1007/s11229-011-9985-6 
Ferreirós, J. (2016), Mathematical Knowledge and the Interplay of Practices, Princeton \& Oxford: Princeton University Press. https://doi.org/10.1515/9781400874002

Ferreirós, J. \& Gray, J. (2006), The Architecture of Modern Mathematics, Oxford: Oxford University Press.

François, K. \& Vandendriessche, E. (2016), 'Reassembling mathematical practices. A philosophical-anthropological approach,' Revista Latinoamericana de Etnomatemática, vol. 9, no. 2, pp. 144-167. https://doi.org/10.22267/relatem.1692.7

Friend, M. (2016), Pluralism in Mathematics: A New Position in Philosophy of Mathematics, Dordrecht: Springer.

Gerovitch, S. (2016), 'Creative discomfort: The culture of the Gelfand Seminar at Moscow University,' In B. Larvor (ed.) Mathematical Cultures. The London Meetings 2012-2014, Basel: Birkhäuser, pp. 51-70. https://doi.org/10.1007/978-3-319-28582-5_4

Gentzen, G. (1935), 'Untersuchungen über das logische Schließen, I,' Mathematische Zeitschrift, vol. 39, no. 1, pp. 176-210. https://doi.org/10.1007/BF01201353

Hamami, Y. \& Giardino, V. (forthcoming), 'Au-Delà des Preuves Formelles: Philosophie de la Pratique Mathématique,' in A. Arana, M. Panza, F. Poggiolesi \& P. Wagner (eds.) Précis de Philosophie des Mathématiques et de le Logique, Editions Ellipses.

HoTT (2013), Homotopy Type Theory: Univalent Foundations of Mathematics. Retrieved from https://arxiv.org/abs/1308.0729 [accessed May 2019]

Inglis, M. \& Aberdein, A. (2014), 'Beauty is not simplicity. An analysis of mathematicians' proof appraisals,' Philosophia Mathematica, vol. 23, no. 1, pp. 87-109. https://doi.org/10.1093/philmat/nku014

Inglis, M. \& Aberdein, A. (2016), 'Diversity in proof appraisal,' in B. Larvor (ed.) Mathematical Cultures, Basel: Birkhäuser, pp. 163-179. https://doi.org/10.1007/978-3-319-28582-5_10

Johansen, M. W.; Misfeldt, M. \& Lomholt Pallavicini, J. (2018), 'A typology of mathematical diagrams,' in P. Chapman, G. Stapleton, A. Moktefi, S. Perez-Kriz \& F. Bellucci (eds.) Diagrammatic Representation and Inference, Proceedings of the 10th International Conference, Diagrams 2018, Edinburgh, UK, June 18-22, 2018, pp. 105-119. https://doi.org/10.1007/978-3-319-91376-6_13

Kaufman, S. (2016), 'On the emergence of a new mathematical object,' in B. Larvor (ed.) Mathematical Cultures. The London Meetings 2012-2014, Basel: Birkhäuser, pp. 91-110.

Lakatos, I. (1978), Philosophical Papers, Vol. I-II, edited by G. Currie and J. Worral, Cambridge: Cambridge University Press.

Lakatos, I. (2015), Proofs and Refutations: The Logic of Mathematical Discovery, Cambridge: Cambridge University Press. https://doi.org/10.1017/CBO9781316286425 
Larvor, B. P. (1997), 'Lakatos as historian of mathematics,' Philosophia Mathematica, vol. 5, no. 1, pp. 42-64. https://doi.org/10.1093/philmat/5.1.42

Larvor, B. (2001), 'What is dialectical philosophy of mathematics?' Philosophia Mathematica, vol. 9, no. 2, pp. 212-229. https://doi.org/10.1093/philmat/9.2.212

Larvor, B. (2008), 'What can the philosophy of mathematics learn from the history of mathematics?' Erkenntnis, vol. 68, no. 3, pp. 393-407. https://doi.org/10.1007/s10670-008-9107-0

Larvor, B. (2010), 'Syntactic analogies and impossible extensions,' in B. Löwe \& T. Müller (eds.) PhiMSAMP_Philosophy of Mathematics: Sociological Aspects and Mathematical Practice, London: College Publications, pp. 197-208.

Larvor, B. (2012), 'How to think about informal proofs,' Synthese, vol. 187, no. 2, pp. 715-730. https://doi.org/10.1007/s11229-011-0007-5

Larvor, B., ed. (2016), Mathematical Cultures: The London Meetings 2012-2014, Basel: Birkhäuser. https://doi.org/10.1007/978-3-319-28582-5

Larvor, B. (2017), 'From Euclidean geometry to knots and nets,' Synthese, pp. 1-22. https://doi.org/10.1007/s11229-017-1558-x

Leitgeb, H. (2009), 'On formal and informal provability,' in O. Bueno \& Ø. Linnebo (eds.) New Waves in Philosophy of Mathematics, London \& New York: Palgrave Macmillan, pp. 263-299. https://doi.org/10.1057/9780230245198_13

Leng, M. (2002), 'Phenomenology and mathematical practice,' Philosophia Mathematica, vol. 10, no. 1, pp. 3-14. https://doi.org/10.1093/philmat/10.1.3

Lillehammer, H. (2014a), 'Who is my neighbour? Understanding indifference as a vice,' Philosophy, vol. 89, no. 4, pp. 559-579. https://doi.org/10.1017/S003181911400028X

Lillehammer, H. (2014b), 'Minding your own business? Understanding indifference as a virtue,' Philosophical Perspectives, vol. 28, no. 1, pp. 111-126. https://doi.org/10.1111/phpe.12037

Löwe, B. (2016), 'Philosophy or not? The study of cultures and practices of mathematics,' in J. Shier, B. Löwe, T. Müller \& Y. Xie (eds.) Cultures of Mathematics and Logic, Basel: Birkhäuser, pp. 23-42. https://doi.org/10.1007/978-3-319-31502-7_2

Löwe, B. \& Müller, T. (2008), 'Mathematical knowledge is context dependent,' Grazer Philosophische Studien, vol. 76, pp. 91-107. https://doi.org/10.1163/9789401206020_005

Löwe, B.; Müller, T. \& Müller-Hill, E. (2010), 'Mathematical knowledge as a case study in empirical philosophy of mathematics,' in B. Van Kerkhove, J. De Vuyst \& J. P. Van Bendegem (eds.) Philosophical Perspectives on Mathematical Practice, London: College Publications, pp. 185-203.

Löwe, B. \& Van Kerkhove, B. (2019), 'Methodological triangulation in empirical philosophy (of mathematics),' in A. Aberdein \& M. Inglis (eds.) Advances in Experimental Philosophy of Logic and Mathematics, London: Bloomsbury, pp. 15-38. 
MacLane, S. (2012), Mathematics: Form and Function, Dordrecht: Springer Science \& Business Media.

Maddy, P. (1988a), 'Believing the axioms I,' The Journal of Symbolic Logic, vol. 53, no. 2, pp. 481-511. https://doi.org/10.1017/S0022481200028425

Maddy, P. (1988b), 'Believing the axioms II,' The Journal of Symbolic Logic, vol. 53, no. 3, pp. 736-764. https://doi.org/10.2307/2274569

Maddy, P. (1997), Naturalism in Mathematics, Oxford: Oxford University Press.

Maddy, P. (2007), Second Philosophy, Oxford: Oxford University Press. https://doi.org/10.1093/acprof:oso/9780199273669.001.0001

Maddy, P. (2011), Defending the Axioms: On the Philosophical Foundations of Set Theory, Oxford: Oxford University Press. https://doi.org/10.1093/acprof:oso/9780199596188.001.0001

Mancosu, P. (2008), The Philosophy of Mathematical Practice, Oxford: Oxford University Press on Demand. https://doi.org/10.1093/acprof:oso/9780199296453.001.0001

Manders, K. (2008), 'The Euclidean diagram,' in P. Mancosu (ed.) The Philosophy of Mathematical Practice, Oxford: Oxford University Press, pp. 80-133. https://doi.org/10.1093/acprof:oso/9780199296453.003.0005

Martin, U. (2015), 'Stumbling around in the dark. Lessons from everyday mathematics,' in A. Felty \& A. Middeldorp (eds.) Automated Deduction: CADE-25, Dordrecht: Springer, pp. 29-51. https://doi.org/10.1007/978-3-319-21401-6_2

Núńez, R. (2005), 'Creating mathematical infinities: Metaphor, blending, and the beauty of transfinite cardinals,' Journal of Pragmatics, vol. 37, no. 10, pp. 1717-1741. https://doi.org/10.1016/j.pragma.2004.09.013

Pantsar, M. (2015), 'Assessing the "Empirical Philosophy of Mathematics",' Discipline Filosofiche, vol. 25, pp. 111-130.

Pitt, J. C. (2001), 'The dilemma of case studies: Toward a Heraclitian philosophy of science,' Perspectives on Science, vol. 9, no. 4, pp. 373-382. https://doi.org/10.1162/106361401760375785

Rav, Y. (1999), 'Why do we prove theorems?' Philosophia Mathematica, vol. 7, no. 1, pp. 5-41. https://doi.org/10.1093/philmat/7.1.5

Rittberg, C. (2016a), 'Mathematical pull,' in B. Larvor (ed.) Mathematical Cultures, Basel: Birkhäuser, pp. 287-302. https://doi.org/10.1007/978-3-319-28582-5_17

Rittberg, C. (2016b), Methods, Goals and Metaphysics in Contemporary Set Theory, PhD thesis, University of Hertfordshire, UK.

Rittberg, C. (2018), 'The Happy Enthusiast,' in B. Van Kerkhove, K. François, S. Ducheyne \& P. Allo (eds.) Laat Ons Niet Ernstig Blijven - Huldeboek voor Jean Paul Van Bendegem, Gent: Academia Press, pp. 83-86.

Rittberg, C. J.; Tanswell, F. S. \& Van Bendegem, J. P. (2018), 'Epistemic injustice in mathematics,' Synthese, Online First. https://doi.org/10.1007/s11229-018-01981-1 
Rittberg, C. J. \& Van Kerkhove, B. (2019), 'Studying mathematical practices: the dilemma of case studies,' ZDM Mathematics Education, Online First. https://doi.org/10.1007/s11858-019-01038-8

Roberts, R. C. \& Wood, W. J. (2007), Intellectual Virtues: An Essay in Regulative Epistemology. Oxford: Oxford University Press on Demand. https://doi.org/10.1093/acprof:oso/9780199283675.003.0001

Rouse, J. (1996), Engaging Science: How to Understand its Practices Philosophically, New York: Cornell University Press.

Schickore, J. (2011), 'More thoughts on HPS: Another 20 years later,' Perspectives on Science, vol. 19, no. 4, pp. 453-481. https://doi.org/10.1162/POSC_a_00049

Scholl, R. \& Räz, T. (2016), 'Towards a methodology for integrated history and philosophy of science,' in T. Sauer \& R. Scholl (eds.) The Philosophy of Historical Case Studies, Boston Studies in the History and Philosophy of Science, vol. 319, Dordrecht: Springer, pp. 69-91. https://doi.org/10.1007/978-3-319-30229-4_5

Soler, L.; Zwart, S.; Lynch, M. \& Israel-Jost, V., eds. (2014), Science after the Practice Turn in the Philosophy, History, and Social Studies of Science, Abingdon: Routledge.

Sørensen, H. K.; Danielsen, K. \& Andersen, L. E. (2019), 'Teacher reader engagement as aspect of proof,' ZDM Mathematics Education, Online First. https://doi.org/10.1007/s11858-019-01056-6

Steiner, M. (1978), 'Mathematical explanation,' Philosophical Studies, vol. 34, no. 2, pp. 135-151. https://doi.org/10.1007/BF00354494

Symonds, J. E. \& Gorard, S. (2010), 'Death of mixed methods? Or the rebirth of research as a craft,' Evaluation \& Research in Education, vol. 23, no. 2, pp. 121-136. https://doi.org/10.1080/09500790.2010.483514

Tanswell, F. (2015), 'A problem with the dependence of informal proofs on formal proofs,' Philosophia Mathematica, vol. 23, no. 3, pp. 295-310. https://doi.org/10.1093/philmat/nkv008

Valitova, R. R. (1998), 'Tolerance: vice or virtue?' Russian Studies in Philosophy, vol. 37, no. 1, pp. 22-27. https://doi.org/10.2753/RSP1061-1967370122

Van Bendegem, J. P. (1988), 'Non-formal properties of real mathematical proofs,' PSA: Proceedings of the Biennial Meeting of the Philosophy of Science Association, no. 1, pp. 249-254. https://doi.org/10.1086/psaprocbienmeetp.1988.1.192992

Van Bendegem, J. P. (1990), 'Characteristics of real mathematical proofs,' Structures in Mathematical Theories: Reports of the San Sebastian International Symposium, September 25-29, 1990, pp. 333-338.

Van Bendegem, J. P. (2014), 'The impact of the philosophy of mathematical practice on the philosophy of mathematics,' in L. Soler, S. Zwart, M. Lynch \& V. Israel-Jost (eds.) Science after the Practice Turn in the Philosophy, History, and Social Studies of Science, Abingdon: Routledge, pp. 215-226. 
van der Ham, I. J.; Hamami, Y. \& Mumma, J. (2017), 'Universal intuitions of spatial relations in elementary geometry,' Journal of Cognitive Psychology, vol. 29, no. 3, pp. 269-278. https://doi.org/10.1080/20445911.2016.1257623

Van Kerkhove, B.; De Vuyst, J. \& Van Bendegem, J. P., eds. (2010), Philosophical Perspectives on Mathematical Practice, London: College Publications.

Weber, K. (2008), 'How mathematicians determine if an argument is a valid proof,' Journal for Research in Mathematics Education, vol. 39, no. 4, pp. 431-459.

Zagzebski, L. T. (1996), Virtues of the Mind: An Inquiry into the Nature of Virtue and the Ethical Foundations of Knowledge, Cambridge: Cambridge University Press. https://doi.org/10.1017/CBO9781139174763

Colin Jakob Rittberg is a postdoctoral researcher (wetenschappelijk medewerker) at the Centre for Logic and Philosophy of Science of the Vrije Universiteit Brussel. He holds a doctorate in philosophy from the University of Hertfordshire, UK, and a Diplom (master's degree) in mathematics from the Westfälische Wilhelms-Universität Münster, Germany. He has used his mathematical background to philosophically engage with contemporary settheoretic practices and is currently focussing on developing a virtue theory of mathematical practices. He has published 'How Woodin changed his mind: new thoughts on the Continuum Hypothesis' (2015) in the Archives for History of Exact Sciences and in joint work with Fenner Tanswell and Jean Paul Van Bendegem on 'Epistemic Injustice in Mathematics' (2019) in Synthese. 